\title{
A stochastic model of human gait dynamics
}

\author{
Yosef Ashkenazy ${ }^{\mathrm{a}, *}$, Jeffrey M. Hausdorff ${ }^{\mathrm{b}}$, Plamen Ch. Ivanov ${ }^{\mathrm{a}, \mathrm{b}}$, \\ H. Eugene Stanley ${ }^{\mathrm{a}}$ \\ ${ }^{a}$ Center for Polymer Studies and Department of Physics, Boston University, Boston, MA 02215, USA \\ ${ }^{\mathrm{b}}$ Beth Israel Deaconess Medical Center, Harvard Medical School, Boston, MA 02215, USA
}

Received 16 August 2002

\begin{abstract}
We present a stochastic model of gait rhythm dynamics, based on transitions between different "neural centers", that reproduces distinctive statistical properties of normal human walking. By tuning one model parameter, the transition (hopping) range, the model can describe alterations in gait dynamics from childhood to adulthood-including a decrease in the correlation and volatility exponents with maturation. The model also generates time series with multifractal spectra whose broadness depends only on this parameter. Moreover, we find that the volatility exponent increases monotonically as a function of the width of the multifractal spectrum, suggesting the possibility of a change in multifractality with maturation.
\end{abstract}

(C) 2002 Elsevier Science B.V. All rights reserved.

PACS: $05.40 .-\mathrm{a} ; 87.90 .+\mathrm{y} ; 87.15 . \mathrm{Aa}$

Keywords: Human gait dynamics; Scaling; Multifractals; Volatility correlations; Stochastic modeling; Maturation

\section{Introduction}

Many physical and physiological processes exhibit complex fluctuations characterized by scaling laws [1-9], some with monofractal [2] and others with multifractal

\footnotetext{
* Corresponding author. Current address: Department of Earth Atmosphere and Planetary Sciences, Massachusetts Institute of Technology, Cambridge, MA 02139, USA.

E-mail address: ashkenaz@wind.mit.edu (Y. Ashkenazy).

URL: http://polymer.bu.edu/ ashkenaz/
} 
behavior $^{1}$ [3]. The origin of these complex fluctuations and the factors that contribute to differences in their behavior are largely unknown. Here we develop a physiologically motivated model that may be helpful in explaining some of the properties that contribute to complex dynamics. We focus on one class of signals - the time series of the inter-stride-intervals (ISI) between successive strides in human gait.

Walking is a voluntary process, but under normal circumstances, stride-to-stride regulation of gait is controlled by the nervous system in a largely automatic fashion [10,11]. Gait is regulated in part by "neural centers" within the cortex and the spinal cord [11]. As a result of time-varying inputs, neural activity at different times are thought to be dominated by different centers, leading to complex fluctuations in the ISI "output". Furthermore, as the central nervous system matures from infancy to adulthood, the interaction between neural centers becomes richer [10].

To understand the underlying regulatory mechanisms of walking, deterministic and stochastic models have been proposed. For example, classic "central pattern generator" models are based on oscillatory neural activity, where the interaction between neural centers helps regulate gait dynamics [12-15]. A stochastic version of a central pattern generator model reproduces certain fractal properties of the ISI series [4]. However, existing models do not explain observed changes in scaling exponents [6], and volatility (magnitude) correlations [16-18] (a new finding reported in the present study) that occur during gait maturation from childhood to adulthood.

We propose a stochastic model consisting of a random walk (RW) on a chain, the elements of which represent excitable neural centers. ${ }^{2}$ A step of the RW between element $i$ and element $j$ represents the "hopping" of the excitation from center $i$ to center $j$. The increase of neural interconnectedness with maturation is modeled by increasing the range of hopping sizes of the RW, since larger hopping sizes will allow exploration of more neural centers. This property mimics one aspect of the increasing complexity of the adult nervous system.

\section{Model}

Previous studies [19] have identified neural centers with pacemaker-like qualities that fire with frequency $f_{i}$, so we represent the network of neural centers by different frequency modes. One mode is activated at a given time $\left(I S I \propto 1 / f_{i}\right)$, and the $f_{i}$ are Gaussian distributed. The model is based on the following assumptions (Fig. 1):

- Assumption (i) is that the $f_{i}$ have finite-size correlations, $\left\langle f_{i} f_{i+\delta}\right\rangle /\left\langle f_{i}^{2}\right\rangle=\mathrm{e}^{-\delta / \delta_{0}}$. We assume finite-size correlations among $f_{i}$ because neighboring neurons are likely

\footnotetext{
${ }^{1}$ Series with long-range power-law correlations can be characterized by their second moment $(q=2)$ scaling exponent. It is possible to find a set of scaling exponents, $\tau(q)$, associated with different moments, $q$ [22,23]. If $\mathrm{d} \tau / \mathrm{d} q$ is constant, the series is monofractal. Otherwise if $\mathrm{d} \tau / \mathrm{d} q$ monotonically decreases with $q$, the signal is multifractal. The width of the multifractal spectrum is the magnitude of this decrease, i.e. $\mathrm{d} \tau(-\infty) / \mathrm{d} q-\mathrm{d} \tau(\infty) / \mathrm{d} q$.

${ }^{2}$ We model one specific type of human locomotion, namely "usual" or "directed" gait. This type of voluntary, goal-directed activity has a large automatic component and usually tends to minimize variability.
} 


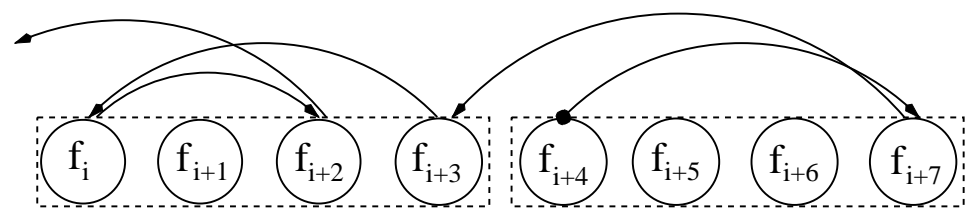

Fig. 1. Illustration of the "neural" hopping model. Shown is a sequence of four transitions, from mode $f_{i+4}$ to $f_{i+7}$ to $f_{i+3}$ to $f_{i}$ to $f_{i+2} \ldots$ Larger values of the hopping-range parameter $C$ expose more modes along the chain. The neuronal zone of size $\delta_{0}=4$ is indicated by the dashed boxes.

to be influenced by similar factors [11]. This assumption effectively creates "neuronal zones" composed of neural centers (modes) along the chain with a typical size $\delta_{0}$.

- Assumption (ii) concerns the rule followed by the RW process. The active neural center is determined by the location of the RW. The hopping sizes of the RW follow a Gaussian distribution of width $C$.

- Assumption (iii) is that a small fraction of noise is added to the output of each mode to mimic biological noise not otherwise modeled. The output $y$ becomes $y(1+A \eta)$ where $A$ is the noise level and $\eta$ is Gaussian white noise with zero mean and unit variance. ${ }^{3}$

The model has three parameters $\delta_{0}, C$, and $A$. We find that the best agreement with the data is achieved when $A=0.02$ and $\delta_{0}=25$. In order to simulate changes with maturation, we vary only the third parameter, $C$, as a function of age, $C=($ age -2$)$ for ages 3-25 years. Increasing the hopping range with age is consistent with the fact that neural transmission is not fully developed until the late teens. ${ }^{4}$

\section{Results}

Examples of ISI time series are shown in Fig. 2a. The ISI series of the adult subject has smaller fluctuations compared with the more variable ISI of the child. Two examples of the model's output are shown in Fig. $2 \mathrm{~b}$; using a large value of $C(C=25)$ simulates the ISI series of an adult, while using a small value $(C=3)$ mimics the ISI

\footnotetext{
${ }^{3}$ It is possible to add the following assumption in order to capture the observed decrease of ISI variability with maturation [6]. When in a mode of frequency $f_{i}$, the model generates as an output $I S I=B / f_{i}$. We suggest the proportionality constant, $B$, to be the ratio between the number of simulated strides and the number of different modes that were visited during a simulation. Then, the standard deviation of the model output decreases when $C$ increases with no effect on the model dynamics.

${ }^{4}$ Central nervous system myelination is not fully complete until the late teens. Consequently, neural processing and the speed of neural transmission do not reach maximum values until this point, consistent with increasing neural interactions with maturation. See Ref. [10]. The model's dynamics can be understood in the following intuitive way. For $C \ll \delta_{0}$ (small hopping range, young child), the dynamics will be confined mainly to a single "neuronal zone"; this will lead to correlations in the ISI time series. For $C \approx \delta_{0}$ (large hopping range, adult), the dynamics will explore frequency modes from different zones; this will lead to a decrease in the correlations. Moreover, for $C \ll \delta_{0}$ the dynamics changes back and forth from one mode to a nearby mode leading to increased magnitude correlations. For $C \approx \delta_{0}$ the dynamics are governed by many different modes leading to the loss of magnitude correlations.
} 


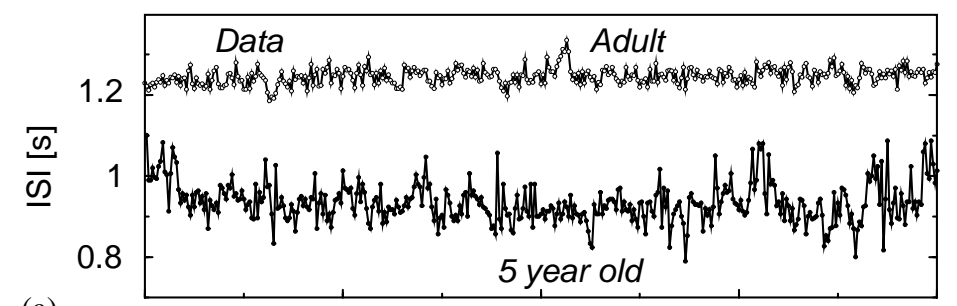

(a)

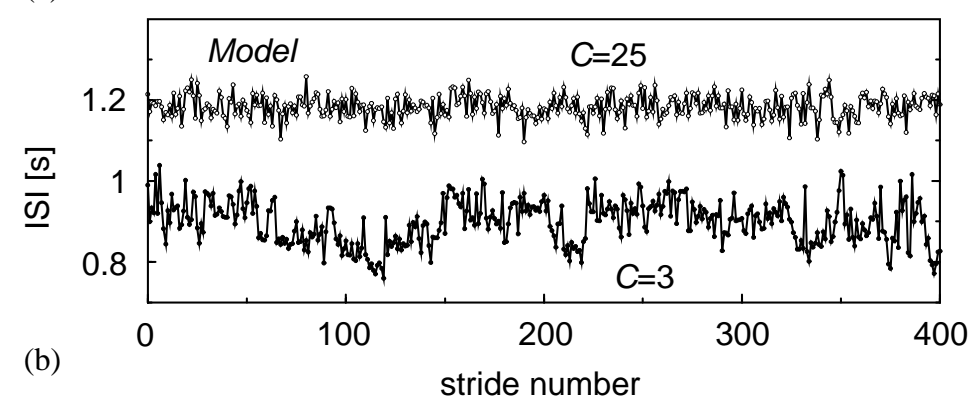

Fig. 2. (a) Examples of ISI series of healthy subjects, ages 5 and 25 years. (b) Examples of ISI generated by the model. Iterating the model with a small value of the hopping-range parameter $(C=3)$ mimics the ISI of young children, while a large value $(C=25)$ mimics that of adults.
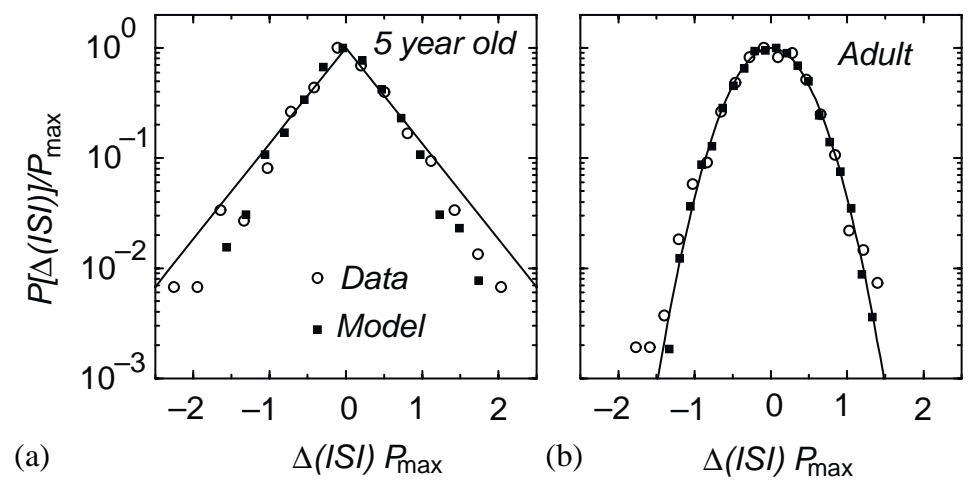

Fig. 3. (a) The normalized distribution of the increments of ISI series $\left(\Delta(I S I)_{i}=(I S I)_{i+1}-(I S I)_{i}\right)$ for the child's ISI series (Fig. 2a) and for the model with $C=3$ (Fig. 2b); both model and data are consistent with an exponential distribution, $P(x)=\mathrm{e}^{-2|x|}$ (solid line). (b) Same as (a) for the adult shown in Fig. 2a and for model $(C=25)$ shown in Fig. $2 b$; in this histogram, both data and model are consistent with a Gaussian distribution, $P(x)=\mathrm{e}^{-\pi x^{2}}$ (solid line).

series of a child. Surprisingly, we find that the normalized probability distributions of the child ISI increment series, $\Delta(I S I)_{i}=(I S I)_{i+1}-(I S I)_{i}$, and of the model with small $C$ are close to exponential (Fig. 3a). In contrast, the distribution converges to Gaussian in the adult and for large $C$ in the model (Fig. 3b).

To further test the model, we study time correlations in the ISI series. We calculate the function $F(n)$, which corresponds to the rms fluctuations of the integrated ISI series of the adult (Fig. 2a) and of the model for $C=25$ (Fig. 2b) [4,5,7]; we use detrended fluctuation analysis for the scaling analysis [20]. Estimating the scaling exponent from 


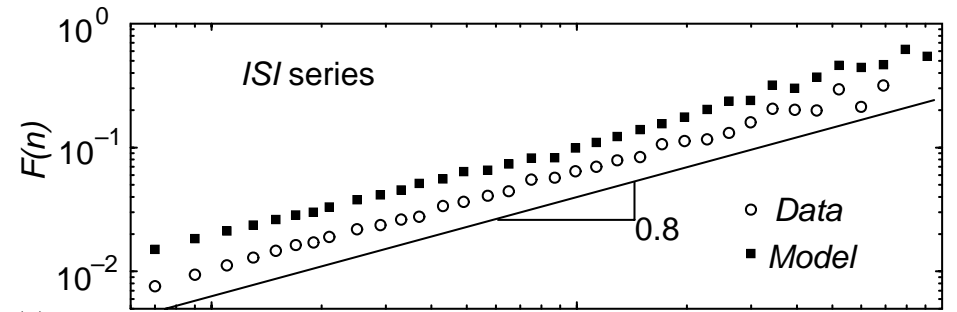

(a)

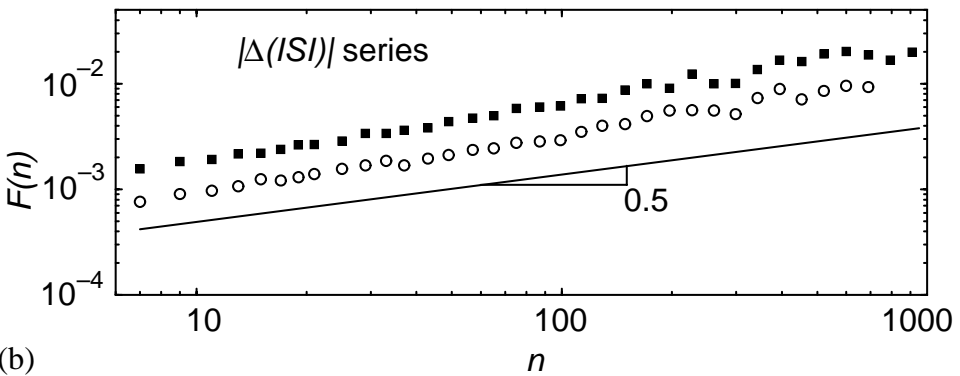

Fig. 4. (a) The rms fluctuation function $F(n)$ for the ISI series of adult $(\bigcirc)$ and of the simulation $(C=25$, $\mathbf{\square})$ shown in Fig. 2. Here $n$ indicates the window size in stride number. Both the data and the model have similar long-range correlation properties for the ISI series. (b) In contrast, $F(n)$ of the magnitudes of the ISI increments $\left|\Delta(I S I)_{i}\right|$ shows weak correlations (scaling exponent of $\approx 0.55$ compared to $\approx 0.5$ of an uncorrelated series).

the slope of $\log F(n)$ vs. $\log n$, we find, for both data and model, that the ISI time series have correlations with scaling exponents $\approx 0.8$ (Fig. $4 \mathrm{a}$ ).

The scaling exponents of the original signal provide an indication of the linear properties (two-point correlations) of the time series. Certain nonlinear aspects may be associated with the presence of long-range correlations in the magnitudes of ISI increments $\left|\Delta(I S I)_{i}\right|$, an index of "volatility" [17,18]. We find (Fig. 4b) that the adult magnitude series is uncorrelated (the scaling exponent is close to 0.5). The model shows similar behavior for large $C$ (Fig. 4b).

Next we inquire if changes in gait dynamics from childhood to adulthood might be reflected in changes in the scaling exponents of the ISI series. The short-range scaling exponents of the ISI series decrease as children mature [6]. We compare the short range scaling exponents for a group of 50 children $^{5}$ [21] with those of 10 adults [5]; this exponent decreases from $\sim 1.0$ to $\sim 0.7$ (Fig. 5a). By altering $C$, the model simulates these maturation-related changes in two-point correlation properties.

The magnitude series exponent of the ISI series also decreases with maturation (Fig. 5b). Our results for the magnitude series suggest that the gait pattern of children is more volatile (and thus more nonlinear) than the usual walking pattern of adults. We note that: (i) unlike adults, young children have difficulty keeping their walking speed

\footnotetext{
${ }^{5}$ Subjects walked at their self-determined, normal pace for $8 \mathrm{~min}$ around a $400 \mathrm{~m}$ running track. Force-sensitive switches were placed inside the subject's right shoe, underneath the heel and the ball of the foot. The force signal was automatically analyzed to determine initial contact time (heel-strike) of each stride. The ISI is the time between consecutive heel-strikes. For more details see Ref. [6].
} 

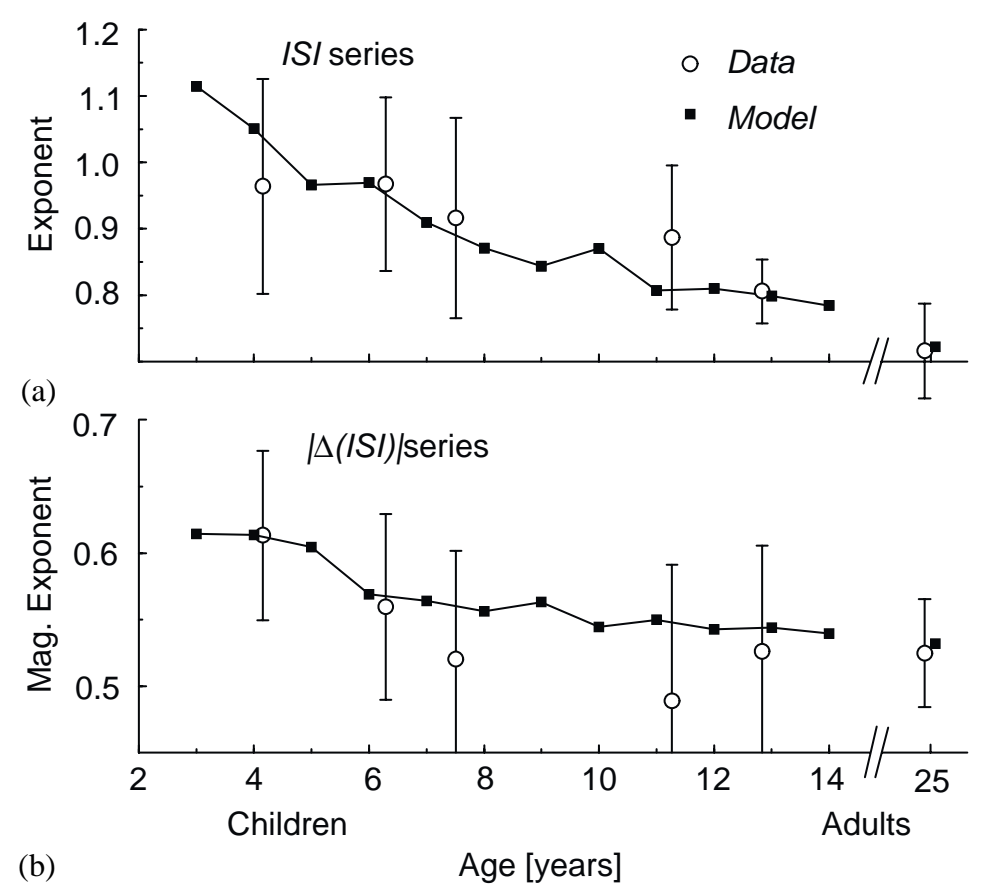

Fig. 5. The scaling exponents of the ISI series of the gait maturation database [21] and for the model. The gait maturation database consists of ISI time series from 50 children between 3 and 13 years old. Each time series is around $8 \mathrm{~min}(\sim 500$ data points $)$. To study the effects of maturation, we divide the database into 5 subgroups: (i) 3-4 year olds (11 subjects), (ii) 5-6 year olds (10 subjects), (iii) 7-8 year olds (14 subjects), (iv) 10-11 year olds (10 subjects), and (v) 12-13 year olds (5 subjects). We also show data [5] from an adult group (10 subjects $1 \mathrm{~h}$ long each; ages 20-30 years). Values for the scaling exponent axis are given as mean \pm standard deviation. For the model simulation, we generate 40 realizations for each value of $C$; the average value is presented (the standard deviation is $\sim 0.05$ ). The age axis for the model follows the relation: age (years) $=C+2$. (a) The short-range scaling exponents of the original ISI series both for the data $(\bigcirc)$ and the model $(\boldsymbol{\square})$. The exponents calculated for window sizes $6<n<13$ steps, decrease with age [6]. The scaling exponent obtained by the model decreases monotonically as $C$ increases and is within the error bars of the data. (b) The scaling exponent of the ISI magnitude series, $\mid \Delta(I S I))_{i} \mid$. The magnitude scaling exponent is calculated for window size $6<n<64$ for the children's group and $6<n<256$ for the adult's group; the maximum window size is $\sim \frac{1}{10}$ of the series length for both groups. The magnitude scaling exponent decreases with age, indicating a loss of magnitude correlations with maturation. The model exhibits a similar decrease and the simulation is within the error bars of the actual data. The subject-to-subject variability is consistent with the scatter observed in physiologic indices of neural development [10].

constant. A large "walking error" in one direction is likely to be followed by a large compensatory walking error in the opposite direction-i.e., a large (or small) increment of ISI is likely to be followed by a large (or small) decrement over a given range of scales. This instability may lead to increased magnitude correlations. (ii) Adults can voluntarily simulate the less stable gait of children and thereby increase the magnitude correlations of the ISI. Children, however, cannot maintain the less volatile dynamics of adults. Thus, in general, the more mature adult gait dynamics are likely to be richer. The model shows (Fig. 5b) a similar decrease of the magnitude exponents when increasing $C$ and is within the error bars of the data. 

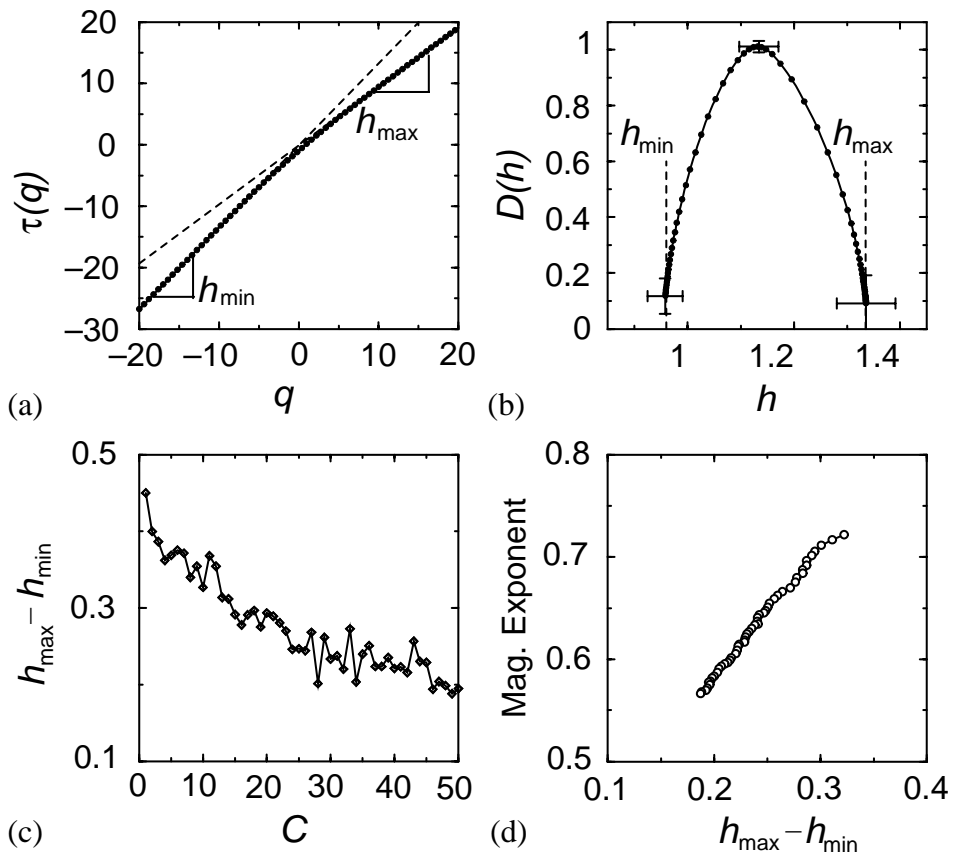

Fig. 6. The multifractal formalism applied to time series generated by the model. For the multifractal analysis, we applied the wavelet transform modulus maxima technique [22,23] with the Daubechies 10-tap discrete wavelet [24] (we repeated the analysis with the continuous Gaussian wavelet transform and obtain similar results). We integrate the series before applying the multifractal formalism. The series length is 32,768 data points and for each value of $C$, we generate 10 realizations; the average value is presented. As the first step of the multifractal analysis we extract the partition function $Z_{q}(n)$ as a function of the window size $n$ for different moments $q$. Then we estimate the scaling exponent $\tau(q)$ from the slope of $\log _{2} Z_{q}(n)$ vs. $\log _{2} n$, where $4 \leqslant n \leqslant 2048$. (a) The exponent $\tau(q)$ vs. the moment $q$ for $C=2$. (b) The Legendre transform $D(h)=q h-\tau(q)$ of (a) where $h(q)=\mathrm{d} \tau / \mathrm{d} q$ and $D(h)$ is the multifractal spectrum. The maximum and minimum Holder exponents, $h_{\max }$ and $h_{\min }$, are indicated by dashed lines and estimated as the slope of $\tau(q)$ for $-20<q \leqslant-15$ and $15 \leqslant q<20$, respectively (see the dashed lines in (a)). Error bars ( 1 standard deviation) are shown for both axes for $q=-20,0$, and 20. (c) The broadness of the multifractal spectrum, $h_{\max }-h_{\min }$, as a function of $C$. For larger $C$, the multifractal spectrum becomes narrower. For $C \gg 1 h_{\max } \sim h_{\min } \sim 0.5$. (d) The scaling exponent of the ISI magnitude series, $\left|\Delta(I S I)_{i}\right|$, increases monotonically as a function of $h_{\max }-h_{\min }$ suggesting an increased multifractality for young children.

The decrease of the magnitude exponent with maturation is consistent with the possibility that the ISI time series becomes less multifractal as the individual matures. This prediction cannot be tested directly on the available data since the time series for the children are too short for multifractal analysis. However, this prediction can be tested indirectly through our model. Fig. 6 summarizes the results of a systematic multifractal analysis on ISI series generated by the model: First, we find narrower multifractal spectra with increasing $C$, suggesting the possibility of a decrease of multifractality with maturation during normal walking (Fig. 6c). Second, we find that the magnitude exponent increases monotonically with the width of the multifractal spectrum (Fig. 6d). These two findings, together with the experimental observation of the decrease of the magnitude exponent with maturation (Fig. 5b), are consistent with the prediction of our 
model of loss of multifractality with maturation. The model's prediction agrees with a recent finding regarding the monofractality of normal gait in adults [25].

\section{Summary}

Before concluding, we note that this relatively simple, stochastic model is unlikely to accurately mirror anatomical structure. However, from a "functional physiology" point of view, the model does capture experimentally observed neurodynamics. While a one-to-one correspondence with neural structures is not likely, the model's behavior should reflect more global features of the neural circuitry that regulates the stride-to-stride fluctuations in gait timing and its changes with maturation and aging. As such, study of the model and its properties may be helpful in elucidating the control of human locomotion.

In summary, we find that a simple stochastic model captures multiple aspects of gait dynamics, and their changes with maturation, including: (i) the shape of the distribution of the ISI increments; (ii) correlation properties of the ISI; and (iii) correlation properties in the magnitudes of the ISI increments. Further, by varying only a single "hopping-range" parameter, $C$, a wide array of multifractal dynamics can be generated. The model can also be altered by "knocking out" certain frequency modes (akin to what may occur during very advanced age or in response to neurodegenerative disease). Simulation with drop-out of frequency modes predicts increased gait variability, with (i) increased magnitude exponents, and (ii) decrease of decreased long-range correlations. Our preliminary analysis of the ISI series of older adults prone to falls is consistent with this prediction. Generalization of the model to two and three-dimensional networks to describe other types of neurological activities is underway.

\section{Acknowledgements}

Partial support was provided by the NIH/National Center for Research Resources (P41 RR13622) NICHD (HD39838), and the NIA (AG14100), P60AG08812. We thank J.J. Collins, S. Havlin, V. Schulte-Frohlinde, C.-K. Peng, and especially to A.L. Goldberger for helpful discussions. Portions of this work were presented at the International Conference on Frontiers In the Physics of Complex Systems, 2001 and appeared in the conference proceeding [Physica A, 302 (2001) 138].

\section{References}

[1] M.F. Shlesinger, Ann. NY Acad. Sci. 504 (1987) 214.

[2] A. Arneodo, E. Bacry, P.V. Graves, J.F. Muzy, Phys. Rev. Lett. 74 (1995) 3293.

[3] P.Ch. Ivanov, L.A.N. Amaral, A.L. Goldberger, S. Havlin, M.G. Rosenblum, Z.R. Struzik, H.E. Stanley, Nature 399 (1999) 461.

[4] J.M. Hausdorff, C.K. Peng, Z. Ladin, J.Y. Wei, A.L. Goldberger, J. Appl. Physiol. 78 (1995) 349.

[5] J.M. Hausdorff, P.L. Purdon, C.K. Peng, Z. Ladin, J.Y. Wei, A.L. Goldberger, J. Appl. Physiol. 80 (1996) 1448. 
[6] J.M. Hausdorff, L. Zemany, C.K. Peng, A.L. Goldberger, J. Appl. Physiol. 86 (1999) 1040.

[7] B.J. West, L. Griffin, Fractals 6 (1998) 101.

[8] J.J. Collins, D.J. DeLuca, Phys. Rev. Lett. 73 (1994) 764.

[9] Y. Chen, M. Ding, J.A.S. Kelso, Phys. Rev. Lett. 79 (1997) 4501.

[10] J.M. Schroder, J. Bohl, U. Vonbardeleben, Acta Neuropathol. 76 (1988) 471.

[11] J.M. Winters, G. Loeb, in: J.M. Winters, P.E. Crago (Eds.), Biomechanics and Neural Control of Posture and Movement, Springer, New York, 2000.

[12] G. Taga, Y. Yamaguchi, H. Shimizu, Biol. Cybern. 65 (1991) 147.

[13] J.J. Collins, I. Stewart, Biol. Cybern. 68 (1993) 287.

[14] M. Golubitsky, I. Stewart, P.L. Buono, J.J. Collins, Nature 401 (1999) 693.

[15] J.H. McAuley, C.D. Marsden, Brain 123 (2000) 1545.

[16] Y. Liu, P. Gopikrishnan, P. Cizeau, M. Meyer, C.K. Peng, H.E. Stanley, Phys. Rev. E 60 (1999) 1390.

[17] Y. Ashkenazy, P.Ch. Ivanov, S. Havlin, C.K. Peng, A.L. Goldberger, H.E. Stanley, Phys. Rev. Lett. 86 (2001) 1900.

[18] Y. Ashkenazy, S. Havlin, P.Ch. Ivanov, C.K. Peng, V. Schulte-Frohlinde, H.E. Stanley, cond-mat/ 0111396, Preprint, 2001.

[19] D. Plenz, S.T. Kital, Nature 400 (1999) 677.

[20] C.K. Peng, S.V. Buldyrev, S. Havlin, M. Simons, H.E. Stanley, A.L. Goldberger, Phys. Rev. E 49 (1994) 1685.

[21] Data are available at http://www.physionet.org/ (Gait Maturation Database).

[22] J.F. Muzy, E. Bacry, A. Arneodo, Phys. Rev. Lett. 67 (1991) 3515.

[23] A. Arneodo, E. Bacry, J.F. Muzy, J. Math. Phys. 39 (1998) 4142.

[24] I. Daubechies, Ten Lectures on Wavelets, SIAM, Philadelphia, 1992.

[25] P.Ch. Ivanov, et al., Unpublished. 